\title{
Dual-frequency VSOP imaging of a high-redshift radio quasar PKS 1402+044
}

\author{
Jun Yang ${ }^{* a, f}$, Leonid Gurvits ${ }^{b}$, Andrei Lobanov ${ }^{c}$, Sándor Frey ${ }^{d, e}$ and Xiao-Yu Hong ${ }^{a}$ \\ ${ }^{a}$ Shanghai Astronomical Observatory of the Chinese Academy of Sciences \\ 80 Nandan Road, Shanghai 200030, P.R. China \\ ${ }^{b}$ Joint Institute for VLBI in Europe, P.O. Box 2, 7990 AA Dwingeloo, the Netherlands \\ ${ }^{c}$ Max-Planck-Institut für Radioastronomie, Auf dem Hügel 69, D-53121 Bonn, Germany \\ ${ }^{d}$ FÖMI Satellite Geodetic Observatory, P. O. Box 585, H-1592 Budapest, Hungary \\ e MTA Research Group for Physical Geodesy and Geodynamics, Budapest, Hungary \\ ${ }^{f}$ Graduate University of the Chinese Academy of Sciences, Beijing 100049, P.R. China \\ E-mail: junyangeshao.ac.cn, Igurvitsejive.nl \\ alobanovempifr-bonn.mpg.de freyesgo.fomi.hy, xhongeshao.ac.ch
}

\begin{abstract}
Based on the VLBI Space Observatory Programme (VSOP) observations at 1.6 and $5 \mathrm{GHz}$, we find that the luminous high-redshift $(z=3.215)$ quasar PKS 1402+044 (J1405+0415) has a pronounced "core-jet" structure. The jet shows a steeper spectral index and lower brightness temperature with the increase of the distance from the core. The variation of brightness temperature is basically consistent with the shock-in-jet model. Assuming that the jet is collimated by the ambient magnetic field, we estimate the mass of the central object as $\sim 10^{9} M_{\odot}$. The upper limit

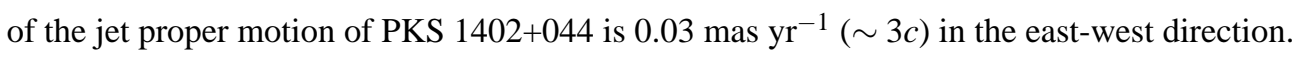

8th European VLBI Network Symposium

September 26-29, 2006

Toruń, Poland

${ }^{*}$ Speaker. 


\section{Introduction}

High-redshift radio quasars can facilitate a comparison of structural properties across the redshift space and provide important inputs into tests of cosmological models, such as the "apparent angular size-redshift" and "apparent proper motion-redshift" relations. PKS 1402+044 (J1405+0415) is a flat-spectrum 0.58-Jy radio source from the Parkes 2.7-GHz survey. Optically, it is a 19.6-magnitude stellar object with the redshift $z=3.215$. MERLIN observations at $1.6 \mathrm{GHz}$ indicated that there is a secondary component at a separation of $0 .{ }^{\prime \prime} 8$ to the south-west at the position angle of $-123^{\circ}$ and a faint extended emission at $3 !^{\prime \prime} 3$ at the position angle of $-106^{\circ}$. VLBI observations at $5 \mathrm{GHz}$ found that the main component consists of a compact core and a resolved jet extending to $\sim 18$ mas to the west [5]. Here, we present some results of VLBI Space Observatory Programme (VSOP) observations at 1.6 and $5 \mathrm{GHz}$. Throughout the paper, the cosmological model with $H_{0}=75 \mathrm{~km} \mathrm{~s}^{-1} \mathrm{Mpc}^{-1}, \Omega_{\mathrm{m}}=0.3$ and $\Omega_{\Lambda}=0.7$ is adopted.

\section{Observations and data reduction}

Using the VSOP space antenna HALCA and the Very Long Baseline Array (VLBA), we observed the radio quasar PKS $1402+044$ in left circular polarization for 8 hours at $1.6 \mathrm{GHz}$ on 21 Jan 2001 and for 7 hours at $5 \mathrm{GHz}$ on 20 Jan 2001. A priori calibrations were done with AIPS in a standard way. Fringes were detected on all space-ground baselines. The useful bandwidth after flagging the side channels spans for $22.8 \mathrm{MHz}$. The imaging, self-calibration and model fitting were done in Difmap.

\section{Results and discussion}
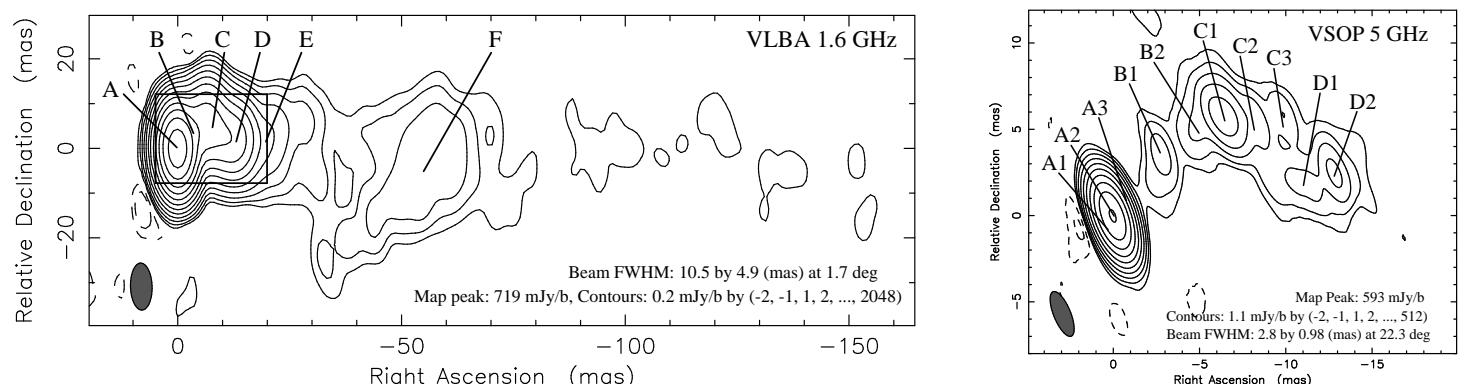

Figure 1: VLBI images of PKS 1402+044 (J1405+0415) at 1.6 and $5 \mathrm{GHz}$. The rectangle in the 1.6-GHz VLBA image shows the area of the 5-GHz VSOP image.

Figure 1 displays a clear core-jet morphology of the quasar PKS 1402+044. The naturally weighted VLBA image detects the weak emission extending up to $\sim 150$ mas $(\sim 1 \mathrm{kpc}$ projected distance). The jet shows a wide section between 20 and 70 mas $(140-500$ pc) indicating an expanding jet propagating in a dense ambient medium. Here, we identify the compact core (component A) and five emission regions (components B - F). The naturally weighted 5-GHz VSOP image shows that the inner jet is resolved into brighter emission regions. With uniform weighting, the jet is basically resolved out. There is a synchrotron self-absorbed weak component (A1) appearing at the beginning of the jet near the brightest component (A2). 
Based on the spectral index image between the two frequencies, we found that the spectral index $\alpha\left(S_{v} \propto v^{\alpha}\right)$ varies from +0.1 in the inner nuclear region to -1.0 in the outer jet regions. To further confirm this variation, we calculated the spectral index of each component using fivefrequency images. The $2.3 / 8.4-\mathrm{GHz}$ data are from the RRFID ${ }^{1}$. The $15-\mathrm{GHz}$ data are from the VSOP support survey by Gurvits et al. (in preparation). The core component has a flat spectrum $\alpha=-0.19$, but the jet components have steeper spectra: $\alpha_{\mathrm{B}+\mathrm{C}}=-0.55, \alpha_{\mathrm{D}}=-0.74$. The spectral difference between the core and jet leads to a decreasing jet-to-core flux density ratio with increasing frequency. Furthermore, this difference demonstrates the explanation of a decreasing jet-to-core flux density ratio at a certain observation frequency with increasing redshift for a large radio quasar sample [3].

If the jet is collimated by the ambient magnetic field $B_{\text {ext }}\left(\sim 10^{-5} \mathrm{G}\right)$ of the host galaxy, the mass of the central object $M_{\mathrm{BH}}$ can be related to the width of the jet $r_{\text {jet }}[1]$ : $M_{\mathrm{BH}} \approx r_{\text {jet }}\left(B_{\mathrm{ext}} / B_{\mathrm{gr}}\right)^{1 / 2}$ $10^{13} M_{\odot}$, where $B_{g r}$ is the magnetic field at the Schwarzschild radius. Based on the theoretical assumption [4], one can expect to have $B_{\mathrm{gr}} \sim 10^{4} \mathrm{G}$. Using the measured size of the component A2 (0.3 mas), the mass of the central object is $\sim 10^{9} M_{\odot}$.

For the radio core, the brightness temperature $T_{\mathrm{B}} \approx 4 \times 10^{12} \mathrm{~K}$ is close to but somewhat larger than the inverse Compton limit. Comparing with the limiting brightness temperature $3 \times 10^{11} \delta^{5 / 6}$ $\mathrm{K}$ in the equipartition jet model of Blandford and Königl [2], a lower limit to the Doppler factor $\delta \approx 22$ can be determined. Following the shock-in-jet model of Marscher [7], we assume that the radio emission is dominated by adiabatic energy losses. The jet plasma has a power-law energy distribution, $N(E) d E \propto E^{-s} d E$. The magnetic field varies as $B \propto d^{-a}$. The Doppler factor is assumed to vary weakly throughout the jet. There is a simple relation: $T_{\mathrm{B}, \text { jet }}=T_{\mathrm{B}, \text { core }}\left(d_{\text {jet }} / d_{\text {core }}\right)^{-\varepsilon}$, where $d_{\text {jet }}$ represents the measured size of core and jet features and $\varepsilon=[2(2 s+1)+3 a(s+1)] / 6$. We take $s=2.5$ and $a=1$ corresponding to the transverse orientation of the magnetic field in the jet [6]. The estimated brightness temperature values are basically consistent with the observed values.

Comparing with another early VLBI observation at $5 \mathrm{GHz}$ in 1986 by Gurvits et al. [5], we

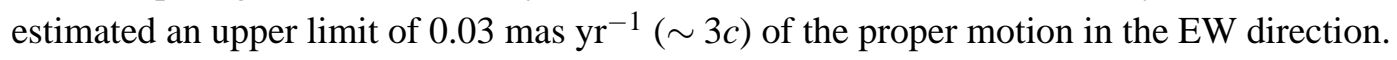

\section{Acknowledgments}

This research was partly supported by the Natural Science Foundation of China (NSFC10473018 and NSFC10333020). Jun Yang and Xiaoyu Hong are grateful to the KNAW-CAS grant. Sándor Frey acknowledges the OTKA T046097 grant. We gratefully acknowledge the VSOP Project, which is led by the Institute of Space and Astronautical Science (Japan) in cooperation with many agencies, institutes and observatories around the world. The National Radio Astronomy Observatory is a facility of the National Science Foundation operated under cooperative agreement by Associated Universities, Inc. This research has made use of NASA's Astrophysics Data System, NASA/IPAC Extragalactic Database (NED) and the United States Naval Observatory (USNO) Radio Reference Frame Image Database (RRFID).

\footnotetext{
${ }^{1}$ USNO Radio Reference Frame Image Database, http://rorf.usno.navy.mil/RRFID
} 


\section{References}

[1] V.S. Beskin 1997, Physi.-Uspekhi, 40(7), 659

[2] R.E. Blandford and A. Königl 1979, ApJ, 232, 34

[3] S. Frey, L.I. Gurvits, K.L. Kellermann et al. 1997, A\&A, 325, 511

[4] G.B. Field and A.D. Rogers 1993, ApJ, 403, 94

[5] L.I. Gurvits, N.S. Kardashev, M.V. Popov et al. 1992, A\&A, 260, 82

[6] A.P. Lobanov, L.I. Gurvits, S. Frey et al. 2001, ApJ, 547, 714

[7] A.P. Marscher 1990, in Parsec-Scale Radio Jets, ed. J. A. Zensus and T. J. Pearson (Cambridge: Cambridge Univ. Press), 236 